\title{
Wide-angle spectral imaging using a Fabry-Pérot interferometer
}

M. Strauch
m.strauch@tudelft.n

\section{L. Livshits}

F. Bociort

\section{H. P. Urbach}

Delft University of Technology, Department of Imaging Physics, Delft, 2628 CH, The Netherlands

CAD Opto-Information and Energy Saving Systems, Saint Petersburg, 199034, Russia

Delft University of Technology, Department of Imaging Physics, Delft, 2628 CH, The Netherlands

Delft University of Technology, Department of Imaging Physics, Delft, 2628 CH, The Netherlands

We show that wide-angle spectral imaging can be achieved with compact and cost-effective devices using Fabry-Pérot interferometers. Designs with a full field of view of $90^{\circ}$, in which the Fabry-Pérot interferometer is mounted either in front of an imaging lens system or behind a telecentric lens system, are presented and analysed. We show the dependency of the spectral resolution on the numerical aperture of the lens system and demonstrate its value as a design criterion.

[DOI: http://dx.doi.org/10.2971/jeos.2015.15037]

Keywords: Multispectral imaging, Fabry-Pérot interferometer, optical design

\section{INTRODUCTION}

Spectral imaging collects spectral information at each image point and thereby enables additional insights in various applications. It is nowadays widely used and many new spectral imaging techniques were developed over the last few years $[1,2]$. While most research focussed on the development of spectral imaging components or on the applications of spectral imaging, we show that there is still potential for the lens design of complete spectral imaging systems.

In this paper we present designs for wide-angle imaging that are simpler and have larger field angles than other known systems. In 2004, Zhang et al. [3] built an interference imaging spectrometer making use of a complex modified Savart polariscope that can reach a full field of view (FOV) of $20^{\circ}$ to $30^{\circ}$ operating in the visible range. Recently Xu et al. [4] published a turntable system using an acousto-optical tunable filter that can scan a full FOV of less than $40^{\circ}$, operating from $450 \mathrm{~nm}$ to $950 \mathrm{~nm}$ with a spectral resolution of $2 \mathrm{~nm}$ to $8 \mathrm{~nm}$. In this paper we will study designs with a full FOV of $90^{\circ}$ that consist only of four components (including the spectral filter). At the same time, more and more applications are found that can benefit from spectral imaging with a large FOV, for example in security and surveillance applications, e.g. for face recognition techniques, that require information in the visible and near infrared range [5, 6]. Target tracing techniques based on spectral imaging [7] were developed and the effects of shadow [8] and darkness [9] and their correction were studied.

In this paper we use a tunable Fabry-Pérot interferometer (FPI) as a spectral filter. The FPI is becoming increasingly popular [10] due to its high light efficiency for a given spectral resolution [11], but it is so far mainly used in remote sensing applications that require high optical resolution, like the Aalto-1 satellite [12] or in drone systems for agricultural observation $[13,14]$ and in telescopes like the vacuum tower telescope on Tenerife [15]. Many new applications do not require such high resolutions, but it is desirable to develop small and cost-effective systems. Following the trend of massproducing small cameras, tunable FPIs can be produced low priced and in large amounts. We will present wide-angle designs of small-size spectral cameras using FPIs and discuss how integrating the FPI characteristics with lens design requirements can improve the performance of FPI based systems.

Section 2 summarises properties of a FPI and relates them to lens design constraints. Section 3 introduces two lens designs, containing a FPI at different positions. Section 4 performs the spectral analysis and shows the impact of the lens design parameters on the FPI performance. The results are summarised in Section 5.

\section{THEORY}

The transmittance of an ideal Fabry-Pérot interferometer (i.e. a FPI illuminated by plane waves without absorption losses) is given by the Airy function

$$
\mathcal{A}(\phi, R)=\frac{1}{1+F(R) \sin ^{2} \frac{\phi}{2}} .
$$

Here the phase $\phi$ depends on the wavelength $\lambda$, the angle of incidence $\theta$ of the incoming rays, the mirror separation $d$ and 
the refractive index $n$ of the medium in between the mirrors as [16]:

$$
\phi=\frac{2 \pi}{\lambda} 2 n d \cos \theta
$$

and $F=4 R /(1-R)^{2}$ is the coefficient of finesse, which depends on the reflectance $R$ of the used mirrors. Maxima are obtained for $\phi=2 \pi m$ for all orders $m \in \mathbb{Z}$. The transmittance function $T$, which takes account of the absorption of the mirrors $A$ can be written as [17]

$$
T(\phi, R, A)=\left(1-\frac{A}{1-R}\right)^{2} \mathcal{A}(\phi, R) .
$$

In the ideal limit of $100 \%$ reflecting mirrors, the transmittance becomes a Dirac comb denoted by the Cyrillic letter Sha: $\amalg(\phi / 2 \pi)$.

For high finesse etalons in practical instruments, the Dirac comb shows different order intensities, that are broadened by convolution with a Lorentzian profile $L\left(x, x_{0}, \gamma\right)$, with the location parameter $x_{0}$ and the half width half maximum $\gamma$. In this case Eq. (3) can be approximated by [18]

$$
\begin{aligned}
& T(\phi) \\
& \quad=\left(\amalg\left(\frac{\phi}{2 \pi}\right) * 2 \pi L(\phi, 0,-\ln (R))\right)\left(1-\frac{A}{1-R}\right)^{2} .
\end{aligned}
$$

With multilayer mirrors using the Bragg effect nearly absorption-free etalons can be made. To calculate the reflectance and transmittance of these mirrors and the air space between them, the standard model that uses a matrix formalism based on the electromagnetic boundary conditions [19] can be employed. Alternatively a model by Gawhary et al. [20], that adds up all internal reflections of the coating can be used, too.

We will investigate two special cases of a spectral imaging device with objects at infinity that contain a Fabry-Pérot interferometer: A collimated configuration, where the FPI is mounted in front of the lens system at a position of collimated incidence, and a telecentric configuration, where the FPI is mounted behind the lens system, that is telecentric on the image side. These two configurations are the easiest way to use a FPI in an existing imaging system with minimal optical redesign. Also, as will be shown below, both choices have their limitations. Using the FPI within the imaging system will lead to designs that will suffer from the limitations of both basic solutions considered here. Eq. (2) is used in both cases to evaluate the situation for plane waves travelling through the FPI.

For collimated incidence the transmittance peak wavelengths depend on the incidence angle and the gap size. The wavefronts coming from an object point are plane waves at the FPI. For plane waves we call the full width half maximum of a transmittance peak produced by the FPI the intrinsic full width half maximum $\mathrm{FWHM}_{\mathrm{i}}$. In this paper we distinguish between the intrinsic plane-wave $\mathrm{FWHM}_{i}$ fully described by Eqs. (1)-(4) and the simulated spherical-wave $F W H M_{s}$, which will be evaluated numerically in the telecentric case.

For the telecentric configuration, the rays coming from a single object point are not parallel at the FPI, i.e. we observe (for a well corrected lens system) a spherical wavefront at the FPI. An estimation of the angle behaviour can be made by considering the plane waves travelling along the marginal and the chief rays. The angle difference between chief ray $\theta_{\text {chief }} \approx 0$ and marginal rays $\theta_{\text {marg }}$ at the FPI broadens the intrinsic transmittance peak of a given order and leads to a decrease in transmittance. To study the design limitation resulting from this effect, we consider the transmittance peak wavelength for incidence $\lambda_{0}$ parallel to the optical axis and the transmittance peak wavelength for marginal incidence $\lambda_{1}=\lambda_{0}-\delta \lambda$. In this paper we focus on only one FPI order. A rough estimation of the design-enforced wavelength interval $\delta \lambda$, within which light can be maximally transmitted, can be derived from Eq. (2) by requiring that the phases for $\lambda_{0}$ and $\lambda_{1}$ are equal

$$
\frac{2 \pi}{\lambda_{0}} 2 n d=\frac{2 \pi}{\lambda_{0}-\delta \lambda} 2 n d \cos \left(\theta_{\operatorname{marg}}\right)
$$

which leads to

$$
\delta \lambda=\left(1-\cos \left(\theta_{\operatorname{marg}}\right)\right) \lambda_{0} .
$$

The wavelength interval can be rewritten in terms of the numerical aperture on the image side $\mathrm{NA}_{\mathrm{i}}=n \sin \left(\theta_{\text {marg }}\right)$ of the corresponding optics:

$$
\delta \lambda=\left(1-\sqrt{1-\left(\frac{\mathrm{NA}_{\mathrm{i}}}{n}\right)^{2}}\right) \lambda_{0}
$$

In air $(n=1)$ this simplifies to

$$
\delta \lambda=\left(1-\sqrt{1-\mathrm{NA}_{\mathrm{i}}^{2}}\right) \lambda_{0} .
$$

Because according to Eq. (6) different wavelengths are associated to the angles between $0^{\circ}$ and $\theta_{\text {marg }}$, the FPI peaks must be broad enough in order to transmit all angles and to avoid a significant decrease in transmittance. A design criterion based on Eq. (6) will be used in the analysis of the model system shown below. As will be shown in Section 4, unless NA is very small, the $\mathrm{FWHM}_{s}$ for spherical waves is proportional to $\delta \lambda$.

\section{MODEL}

The lens model used in this paper is a modified version of a wide-angle pinhole lens with a full FOV of $90^{\circ}$ [21]. The system is smaller than $15 \mathrm{~mm}$ in length and the pinhole has a diameter of $1 \mathrm{~mm}$. The model is analysed with CODE V. With a FPI mounted at two different positions, the two designs were reoptimised to correct the aberrations introduced by the FPI using the default CODE V merit function.

The Fabry-Pérot interferometer is modelled as two planeparallel glass plates having each $0.5 \mathrm{~mm}$ in thickness. The mirror coatings and the air gap are modelled as a coating in between the glass plates (Figure 1). The layer of air in between can be tuned in thickness. The transmittance of the FPI can be tuned by varying the thickness of the air layer in the coating model (Figure 2).

The dimensions of the Fabry-Pérot interferometer are similar to those of commercially available FPIs [10, 22, 23]. For these 


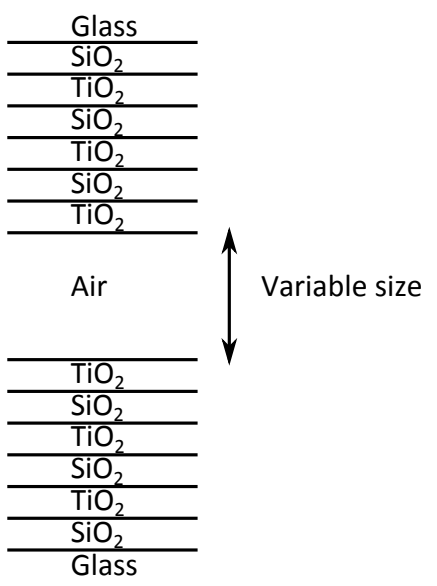

FIG. 1 The coating of the FPI is a quarter wavelength optical thickness stack of $\left(\mathrm{SiO}_{2} \mathrm{TiO}_{2}\right)_{3}$ with a variable air layer.

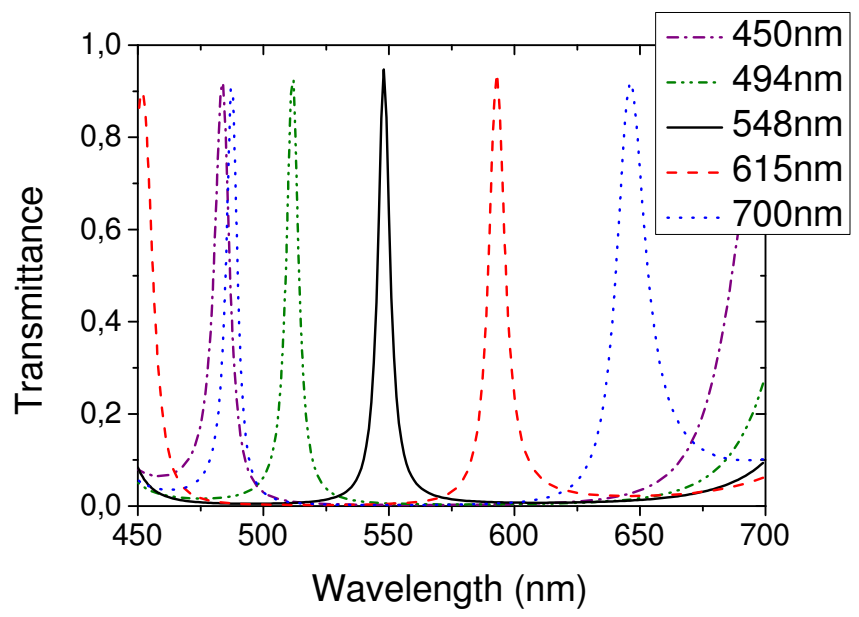

FIG. 2 The modelled FPI transmittance is shown for different air gap sizes in the case of normal incidence.

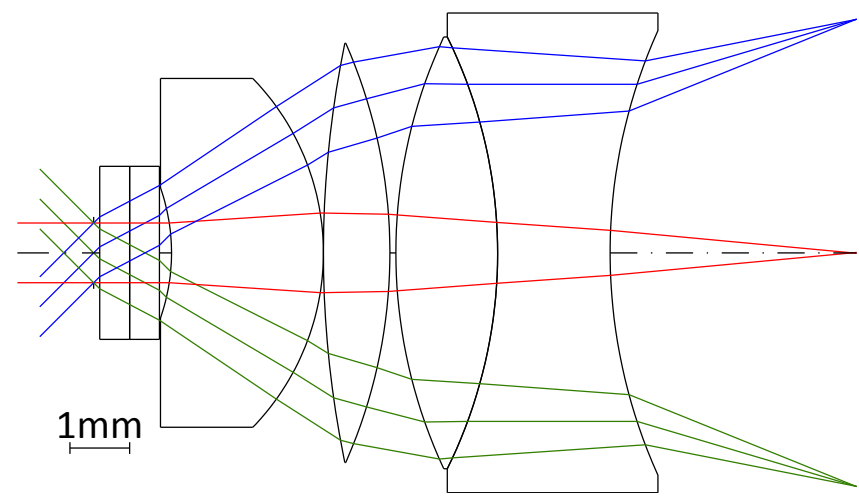

FIG. 3 Wide-angle security lens design with an etalon in collimated configuration. The effective focal length is $5.43 \mathrm{~mm}$ and the numerical aperture on the image side is $\mathrm{NA}_{\mathrm{i}}=0.0927$.

FPIs, aperture diameters are available up to $20 \mathrm{~mm}$ and the air gap can be tuned with sub-nanometre accuracy in just a few milliseconds. To simulate such an FPI, we choose the coating of the mirrors to be a quarter wavelength optical thickness stack, at reference wavelength $548 \mathrm{~nm}$, of a high and a low refractive index material, made of well-understood FPI coating layers $\left(\mathrm{SiO}_{2} \mathrm{TiO}_{2}\right)_{3}$ [24]. The system is evaluated both using the etalon at a position of collimated (Figure 3 ) and telecentric (Figure 4) incidence.

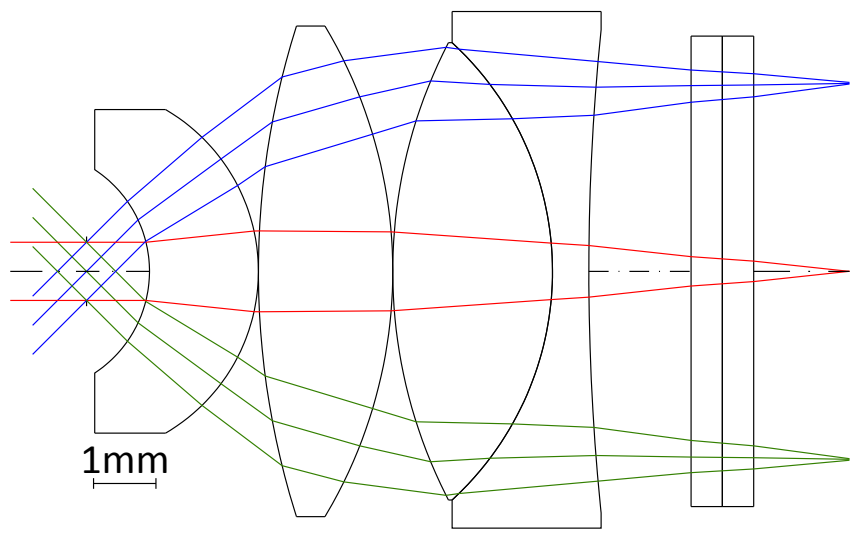

FIG. 4 Wide-angle security lens design an etalon in telecentric configuration. The effective focal length is $4.33 \mathrm{~mm}$ and the numerical aperture on the image side is $N A_{i}=0.108$.

\section{RESULTS}

Figure 5 shows the image quality of the two designs simulated with CODE V. Note that, despite of the large field, the small size and the simplicity of the design, the imaging quality of the system is sufficient for applications such as e.g. surveillance. In CODE V image simulation the input file must be a RGB bitmap, so we can extract only three color channels from the image. Despite of this spectral limitation the simulation provides useful information on the spectral behaviour of the two camera models. The distortion of the images is typical for wide-angle camera systems and can be corrected by software [25]. The image irradiance decreases with the field angle. However, the aperture stop situated in front of the system produces pupil aberrations that lead to a less significant drop than the one expected according to the cosine-fourth law [26]. The different spectral behaviour of the two designs can be best explained by analysing the field angle dependency of the spectral transmittance.

For the collimated design, note that the dominant colors in the centre and at maximal field are different, in accordance with the transmittance given in Figure 6. The Airy function (Eq. (1)) describes the small angle behaviour, visible as a wavelength shift of the peak for $15^{\circ}$ with respect to $0^{\circ}$, without changing the shape of the peak. For large angles, the reflectance of the mirror coatings becomes polarisation dependent and must be described by matrix-based calculations of the thin-film transmittance [19]. For the mirror coating we use, the transmittance is significantly decreasing for field angles larger than $20^{\circ}$. The transmittance peak becomes a superposition of the transmittance peaks of s- and p-polarised light, which makes the retrieval of the spectral information more complex.

The field-dependent transmittance of the telecentric design (Figure 4) is shown in Figure 7. The transmittance and the simulated spectral resolution $\left(\mathrm{FWHM}_{\mathrm{S}}\right)$ remain essentially the same over the entire FOV. Therefore in Figure 5(c), (e), (g) we see only a single color (i.e. wavelength peak) for a given value of the FPI air gap. As for collimated incidence, changing the coating is mainly influencing the $\mathrm{FWHM}_{\mathrm{s}}$ of the transmittance peaks. By increasing the number of coating layers (Figure 8), the mirror reflectance increases and the resulting $\mathrm{FWHM}_{\mathrm{s}}$ decreases. However, when the peaks become too 


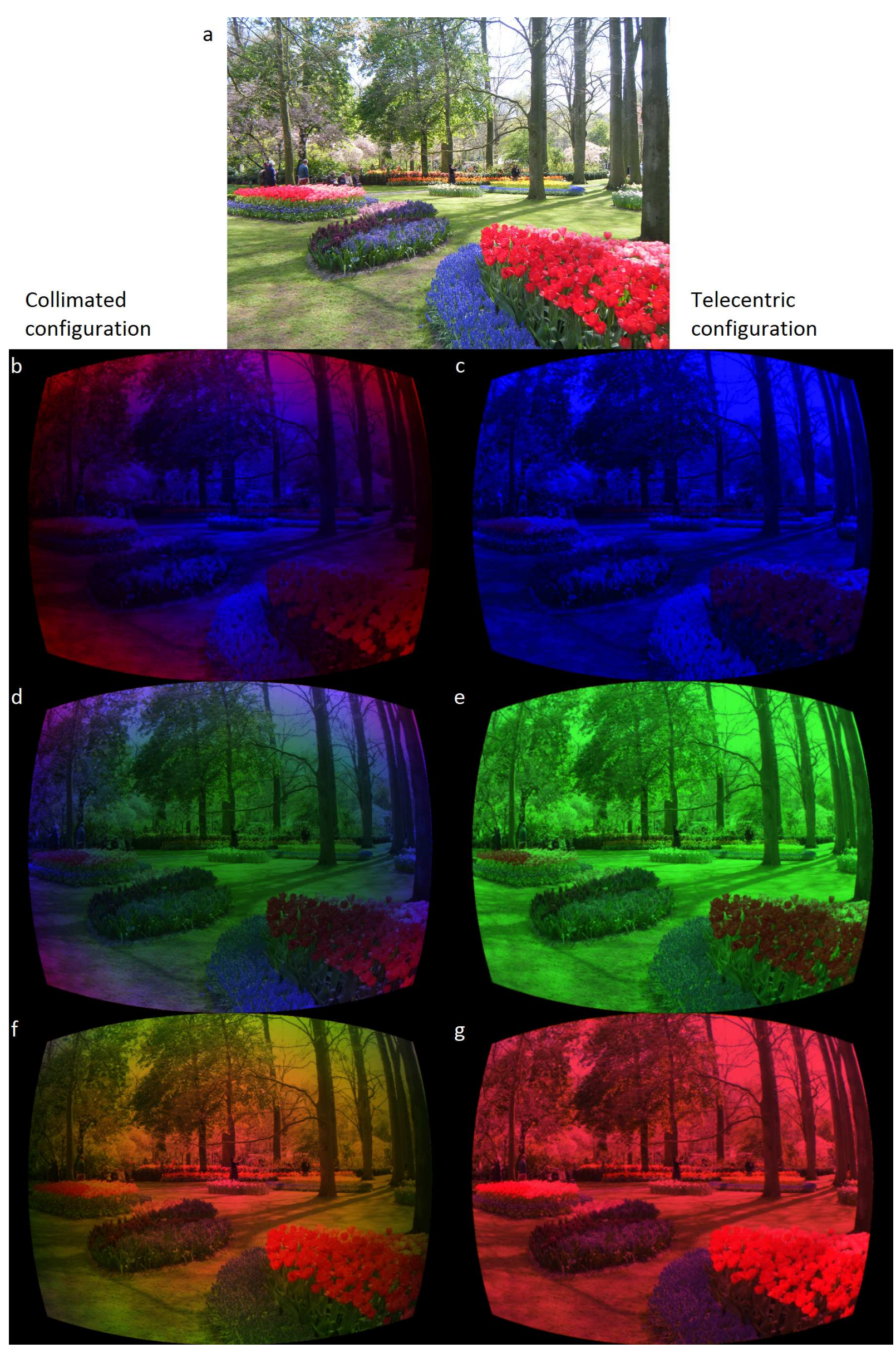

FIC. 5 CODE V image simulation, that shows the spectral behaviour of the collimated (left) and the telecentric configuration (right). The object (a) is a RGB file, so we can only extract information for 3 wavelengths. The images (b) at (c) show the transmittance image for a FPl air gap of $d=190 \mathrm{~nm}$, (d) a (e) for $d=274 \mathrm{~nm}$ and (f) $\mathfrak{\mathrm { t }}$ ( $\mathrm{g}$ ) for $d=360 \mathrm{~nm}$. 


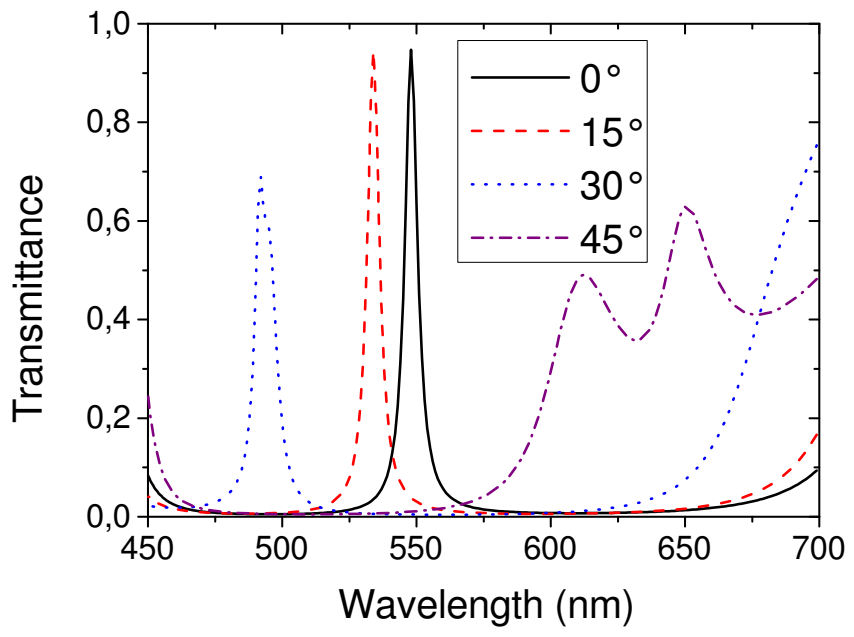

FIG. 6 Transmittance spectra of the collimated configuration (Figure 3) for different half field angles using unpolarised light.

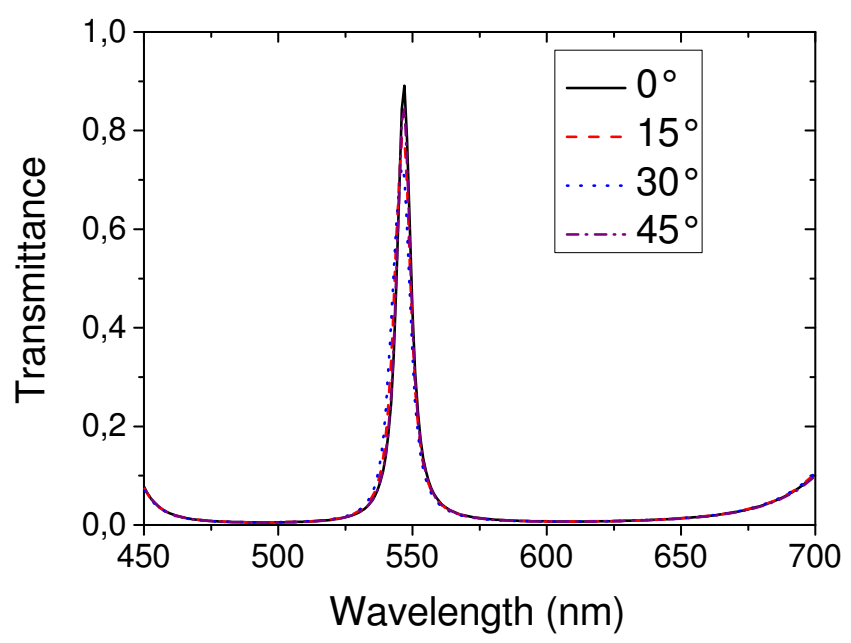

FIG. 7 Transmittance spectra of the telecentric configuration (Figure 4) for different half field angles

narrow to transmit all angles according to Eq. (6), the transmittance starts decreasing significantly. For $\mathrm{NA}_{\mathrm{i}}=0.108$ as used in the telecentric system (Figure 4 ) and $\lambda=548 \mathrm{~nm}, \delta \lambda$ is $3.2 \mathrm{~nm}$. As shown in Figure 8, this is in good agreement with the CODE V transmission analysis for different coatings. In CODE $\mathrm{V}$ all calculations for the transmission analysis are made relative to unit energy across the entrance pupil [27].

By changing the numerical aperture the dependency of the FWHM $_{\mathrm{s}}$ on the numerical aperture can be observed directly (Figure 9). For low numerical apertures, i.e. small wavelength intervals $\delta \lambda$ the simulated $\mathrm{FWHM}_{\mathrm{S}}$ does barely differ from the intrinsic $\mathrm{FWHM}_{\mathrm{i}}$ and is determined by the coating, while for larger numerical apertures the $\mathrm{FWHM}_{\mathrm{S}}$ of the peak becomes proportional to the calculated wavelength interval $\delta \lambda$. This proportionality comes from the fact that when $\mathrm{NA}_{i}$ increases, the necessary $\mathrm{FWHM}_{\mathrm{S}}$ that is required to transmit larger angles also increases. Due to this proportionality Eq. (8) can be used as a criterion to choose a coating or design for a certain spectral resolution as well as to predict the $\mathrm{FWHM}_{\mathrm{S}}$ of a system with a given FPI when the aperture is changed. We can distinguish two domains: For small values of the $\mathrm{NA}_{\mathrm{i}}$, a higher FPI finesse yields a higher spectral resolution without influencing the peak transmittance. For large $\mathrm{NA}_{\mathrm{i}}$, a higher

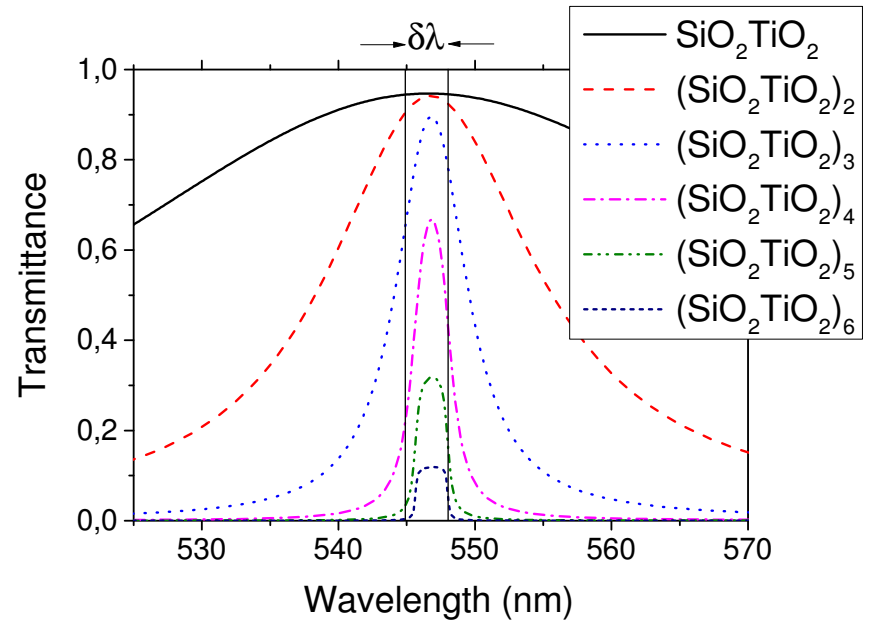

FIG. 8 Transmittance of the telecentric configuration at $0^{\circ}$ half field angle for different numbers of pairs of $\left(\mathrm{SiO}_{2} \mathrm{TiO}_{2}\right)$ as coatings. The wavelength interval $\delta \lambda$ (Eq. (8)) is indicated in the graph, the first vertical line is the peak wavelength for marginal rays $\lambda_{1}$ and the second line is the peak wavelength for the chief ray $\lambda_{0}$.

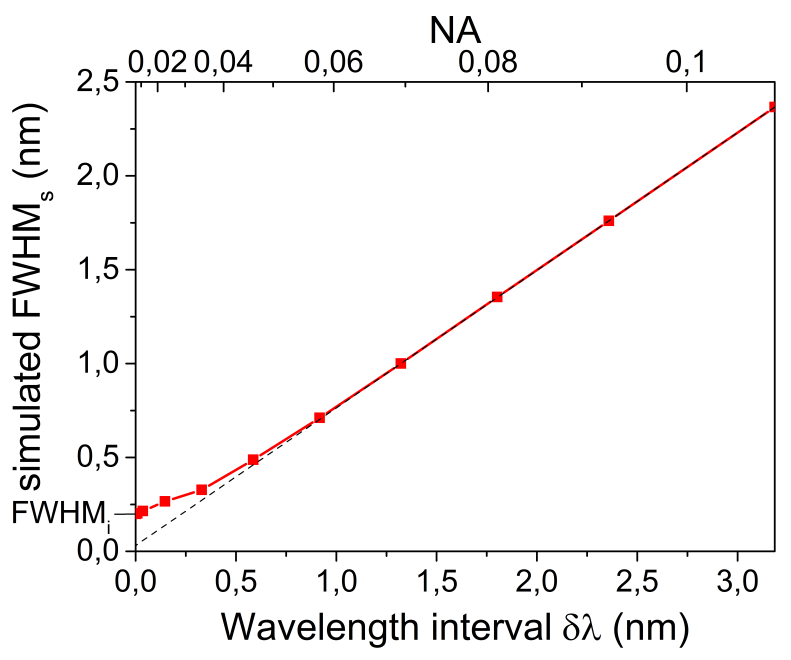

FIC. 9 Simulated $\mathrm{FWHM}_{\mathrm{S}}$ of transmittance peaks in telecentric configuration using a FPI with a pair of $\left(\mathrm{SiO}_{2} \mathrm{TiO}_{2}\right)_{6}$ coatings for different numerical apertures. The first point for $\mathrm{NA}_{\mathrm{i}}=0$ is the intrinsic $\mathrm{FWHM}_{\mathrm{i}}$. For larger numerical apertures the simulated $\mathrm{FWHM}_{\mathrm{S}}$ of the peaks is proportional to the wavelength interval $\delta \lambda$ as indicated by the dotted asymptote.

FPI finesse does not influence the $\mathrm{FWHM}_{\mathrm{S}}$ but the transmittance decreases.

The comparison of the two designs shows their advantages and disadvantages. For the collimated design, the spectral resolution is not limited by the $\mathrm{NA}_{i}$ of the camera design, but digital imaging processing is required. If a hyperspectral image is desired (i.e. many wavelengths are required), the small-angle dependency of the peak transmittance can be corrected by computationally associating all field angles to their corresponding wavelengths. Multispectral imaging, i.e. the case when only few wavelengths must be determined, also requires a full wavelength scan to obtain the necessary information over the full field of view and is therefore as time-consuming as hyperspectral imaging. For large field angles the transmittance peaks are formed by superposition of s- and p-polarised light. Due to the polarisation dependency of the mirror reflectance the peaks are broadened, deformed and even split up into two peaks. The polarisation- 
deformed transmittance for large field angles can only be used if the measured data is deconvolved afterwards on the computer.

In the telecentric configuration, the spectrum transmitted by the FPI is the same for all field angles and independent of polarisation over the full field of view. For multispectral imaging no complete wavelength scan is required, because full FOV information for individual wavelengths can be collected separately. Therefore in the telecentric case we can effectively make use of the advantage of an FPI, that one can select the measured wavelength rapidly, based on the target and goal of the measurements. The width of the transmittance peaks in the telecentric configuration is limited by the numerical aperture on the image side of the imaging optics (Eq. (8)). For a required width of the peaks, the criterion limits the $\mathrm{NA}_{i}$ and therefore the amount of light that can be collected in a given time. Unless high spectral resolution is required the telecentric design is preferable because of its simplicity. For many applications e.g. for security purposes the spectral resolution should be sufficient.

Tighter tolerances of collimated configurations also motivated e.g. Kentischer et al. [15] (who describe a small field design) to use a FPI in a telecentric configuration. They describe how misalignment and large-scale variations of the FPI mirror shape cause only a small wavelength shift in telecentric designs, while the resolution drops significantly in collimated configuration. Manufacturing a FPI with flat and parallel mirrors, which do not bend when the gap size or environmental parameters are changed, is challenging. The price of a FPI therefore scales with the quality of its mirrors, therefore the less restrictive tolerances make the telecentric configuration more cost-effective for multispectral imaging systems than the collimated one. The telecentric system (Figure 4) can be built by choosing an appropriate existing FPI and by rescaling the imaging part of the system accordingly. We expect our design to be useful in situations where the scene is illuminated sufficiently.

\section{CONCLUSIONS}

In this paper we show that for a multispectral imaging system a large field of view can be achieved with a simple and compact design. While the common approach of inserting the FPI in front of the lens system is not able to produce a large field of view, the telecentric design shown in Figure 4 can achieve this. As shown in Eq. (8) and numerically confirmed with CODE V (see Figure 9) the spectral resolution can be increased by decreasing the aperture size at the expense of the amount of collected light at the detector. This property can be used as a design criterion for telecentric multispectral imaging systems. Depending on the numerical aperture $\mathrm{NA}_{i}$, we find two domains. In the first case, increasing the FPI finesse has the desirable effect of increasing the spectral resolution. In the second case, increasing the FPI finesse has the negative effect of decreasing the transmittance. Unless high spectral resolution is required, because of its simplicity and its reasonable imaging quality this design has the potential to be used in many applications like e.g. surveillance camera systems. De- sign strategies that are similar to the ones shown here can be applied in other design tasks as well, for instance in the near infrared spectral region.

\section{ACKNOWLEDGEMENTS}

We acknowledge the use of an educational licence of CODE V. We kindly thank Luca Cisotto for providing us with the photo of the tulips at Keukenhof. This work is funded through the Spectr@phone project (IPD 12017) of the IOP Photonic Devices program of RVO.

\section{References}

[1] N. Hagen, and M. W. Kudenov, "Review of snapshot spectral imaging technologies," Opt. Eng. 52, 090901 (2013).

[2] R. C. Lyon, D. S. Lester, E. N. Lewis, E. Lee, L. X. Yu, E. H. Jefferson, and A. S. Hussain, "Near-infrared spectral imaging for quality assurance of pharmaceutical products: Analysis of tablets to assess powder blend homogeneity," AAPS PharmSciTech. 3, 1-15 (2002).

[3] C. Zhang, B. Zhao, and B. Xiangli, "Wide-field-of-view polarization interference imaging spectrometer," Appl. 0pt. 43, 6090-6094 (2004).

[4] Y. Xu, R. Xu, F. Li, and J. Wang, "Verification of programmable, large-FOV spectral imaging technology based on a staring/scanning area-array detector," Proc. SPIE 9263, 92630l (2014).

[5] Z. Pan, G. Healey, M. Prasad, and B. Tromberg, "Face recognition in hyperspectral images," IEEE T. Pattern Anal. 25, 1552-1560 (2003).

[6] H. Chang, A. Koschan, M. Abidi, S. C. Kong, and C.-H. Won, "Multispectral visible and infrared imaging for face recognition," in Proceedings to the IEEE Computer Society Conference on Computer Vision and Pattern Recognition Workshops, 1-6 (IEEE, Anchorage, 2008).

[7] P. J. Nystrom, and L. K. Mestha, "Automatically focusing a spectral imaging system onto an object in a scene," US20140240511 A1 (2014).

[8] C. Feng, X. Zhang, L. Shen, and S. Zhuo, "Multi-spectral imaging system for shadow detection and attenuation," US20140240477 A1 (2014).

[9] H. Maeng, S. Liao, D. Kang, S.-W. Lee, and A. K. Jain, “Nighttime face recognition at long distance: Cross-distance and crossspectral matching," in Computer Vision - ACCV 2012, K. M. Lee, Y. Matsushita, J. M. Rehg, and Z. Hu, eds., 708-721 (Springer Berlin Heidelberg, 2013).

[10] J. Antila, R. Mannila, U. Kantojärvi, C. Holmlund, A. Rissanen, I. Näkki, J. Ollila, and H. Saari, "Spectral imaging device based on a tuneable MEMS Fabry-Perot interferometer," Proc. SPIE 8374, $83740 \mathrm{~F}$ (2012).

[11] P. Jacquinot, "The luminosity of spectrometers with prisms, gratings, or Fabry-Pérot etalons," J. Opt. Soc. Am. 44, 761-765 (1954).

[12] J. Praks, A. Kestilä, M. Hallikainen, H. Saari, J. Antila, P. Janhunen, and R. Vainio, "Aalto-1 - An experimental nanosatellite for hyperspectral remote sensing," in Proceedings to the IEEE International Geoscience and Remote Sensing Symposium (IGARSS), 4367-4370 (IEEE, Vancouver, 2011). 
[13] H. Saari, I. Pölönen, H. Salo, E. Honkavaara, T. Hakala, C. Holmlund, J. Mäkynen, R. Mannila, T. Antila, and A. Akujärvi, "Miniaturized hyperspectral imager calibration and UAV flight campaigns", Proc. SPIE 8889, 888910 (2013).

[14] E. Honkavaara, J. Kaivosoja, J. Mäkynen, I. Pellikka, L. Pesonen, H. Saari, H. Salo, T. Hakala, L. Marklelin, and T. Rosnell, “Hyperspectral reflectance signatures and point clouds for precision agriculture by light weight UAV imaging system", ISPRS Ann. Photogramm. Remote Sens. Spatial Inf. Sci. I-7, 353-358 (2012).

[15] T. J. Kentischer, W. Schmidt, M. Sigwarth, and M. v. Uexküll, "TESOS, a double Fabry-Perot instrument for solar spectroscopy," Astron. Astrophys. 340, 569-578 (1998).

[16] E. Hecht, Optics (fourth edition, Addison Wesley, San Francisco, 2002).

[17] J. M. Vaughan, The Fabry-Perot interferometer - History, theory, practice and applications (IOP Publishing Ltd, Bristol, 1989).

[18] W. H. Steel, Interferometry (Cambridge University Press, New York, 1967).

[19] H. A. Macleod, Thin-film optical filters (fourth edition, CRC Press, Boca Raton, 2010).

[20] 0. El Gawhary, M. C. Dheur, S. F. Pereira, and J. J. M. Braat, "Extension of the classical Fabry-Perot formula to $1 \mathrm{D}$ multilayered structures," Appl. Phys. B 111, 637-645 (2013).
[21] I. Livshits, Z. Hou, P. van Grol, Y. Shao, M. van Turnhout, P. Urbach, and F. Bociort, "Using saddle points for challenging optical design tasks," Proc. SPIE 9192, 919204 (2014).

[22] http://www.rikola.fi/site/?page_id=46.

[23] http://goochandhousego.com/news/ miniature-spectral-imaging-camera/

[24] B. Schmitt, J. P. Borgogno, G. Albrand, and E. Pelletier, "In situ and air index measurements: influence of the deposition parameters on the shift of $\mathrm{TiO}_{2} / \mathrm{SiO}_{2}$ Fabry-Perot filters," Appl. Opt. 25, 3909-3915 (1986)

[25] D. C. Brown, "Decentering distortion of lenses," Photogramm. Eng. 32, 444-462 (1966).

[26] M. Aggarwal, H. Hua, and N. Ahuja, "On cosine-fourth and vignetting effects in real lenses," Proceedings to the Eighth IEEE International Conference on Computer Vision, 472-479 (IEEE, Vancouver, 2001).

[27] CODE V Systems Analysis Reference Manual 10.6 (Synopsys, 2013). 